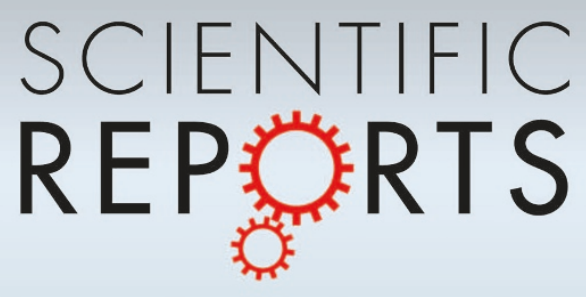

OPEN

SUBJECT AREAS:

ENZYME MECHANISMS

SINGLE-MOLECULE BIOPHYSICS

Received

8 April 2014

Accepted

21 August 2014

Published

29 September 2014

Correspondence and requests for materials should be addressed to S.M. (smyong@illinois. edu)

\title{
Single-molecule real-time detection of telomerase extension activity
}

\author{
Helen Hwang ${ }^{1}$, Patricia Opresko² \& Sua Myong ${ }^{1,3,4,5}$
}

\begin{abstract}
'Bioengineering Department, University of Illinois, ${ }^{2}$ Department of Environmental and Occupational Health, University of Pittsburgh, ${ }^{3}$ Biophysics and Computational Biology, University of Illinois, ${ }^{4}$ Institute for Genomic Biology, University of Illinois, ${ }^{5}$ Physics Frontier Center (Center of Physics for Living Cells), University of Illinois.
\end{abstract}

The ends of eukaryotic chromosomes are capped by telomeres which consist of tandem G-rich DNA repeats stabilized by the shelterin protein complex. Telomeres shorten progressively in most normal cells due to the end replication problem. In more than $85 \%$ of cancers however, the telomere length is maintained by telomerase; a reverse transcriptase that adds telomeric TTAGGG repeats using its integral RNA template. The strong association between telomerase activity and malignancy in many cancers suggests that telomerase activity could serve as a diagnostic marker. We demonstrate single-molecule, real-time telomerase extension activity observed digitally as the telomeric repeats are added to a substrate. The human telomerase complex pulled down from mammalian cells displays extension activity dependent on dNTP concentration. In complex with the processivity factor, POT1-TPP1, telomerase adds repeats at an accelerated rate and yields longer products. Our assay provides a unique detection platform that enables the study of telomerase kinetics with single molecule resolution.

.

he telomerase holoenzyme consists of the telomerase catalytic subunit (hTERT), the telomerase RNA $(\mathrm{hTR})^{1-3}$, dyskerin ${ }^{4-6}$ and TCAB1 ${ }^{7}$. Early methods developed for measuring telomerase activity include the primer extension assay ${ }^{8}$ and the widely used telomeric repeat amplification protocol (TRAP) ${ }^{9-12}$. Due to the limitations of TRAP assay resulting from potential PCR induced errors, a more quantitative Q-TRAP assay, advanced primer extension method, optically based detection and electrochemical strategies have been devised to measure telomerase activity in ensemble biochemistry assays (see review ${ }^{13}$ for more complete list). Ren et al developed a single molecule assay whereby the Alexa 488 labeled DNA template and Cy5-dATP were applied to single units of telomerase. The accumulated increase of Cy5 intensity was measured and converted to the telomerase extension activity ${ }^{14}$.

To obtain a direct and digital readout of the telomeric extension, we developed a single molecule telomerase assay in which telomeric repeat addition is detected as a discrete and stepwise signal increase in real time. This platform enables one to directly measure the rate at which a telomeric substrate is extended by a single unit of telomerase alone or in conjunction with other proteins such as the shelterin components. It also allows us to quantitate the total length a telomeric overhang is extended by a single telomerase complex in one cycle of binding. Using this detection stage, we demonstrate that telomerase activity involves an initial activation period that precedes an active phase of extension. Both the activation and extension rates show a dependence on the dNTP concentration. Remarkably, in complex with the two shelterin components, POT1-TPP1, telomerase extends at a faster rate and generates longer telomeric products. Taken together, these data provide a molecular basis by which POT1-TPP1 increases the processivity of telomerase ${ }^{15}$. Further, we show that this increased processivity may arise from the dynamic sliding of POT1-TPP1 that induces fast translocation of telomerase.

\section{Results}

Telomerase extension activity visualized as discrete steps. To obtain telomerase, HEK 293 cells were transfected with flag-tagged telomerase overexpression plasmids (generous gift from Gaël Cristofari and Tom Cech) ${ }^{16}$. We applied the cell lysate to a single molecule imaging surface pretreated with anti-flag antibody to pull down flagtagged telomerase ${ }^{17}$. We visualized the captured telomerase by using a primary antibody against telomerase and fluorescently labeled secondary antibody and further checked the pull-down efficiency and specificity by control experiments as reported recently ${ }^{18}$. We determined the lysate concentration to achieve the appropriate single molecule density of telomerase on the imaging surface. To visualize telomeric DNA binding to telomerase, we applied a fluorescently labeled DNA substrate that possesses three repeats of the telomeric overhang, (TTAGGG) which should anneal to the telomeric RNA (hTR). We confirmed the binding of DNA to telomerase by 
colocalization $^{18}$. One field of view yields about 60-80 molecules of DNA bound telomerase (Supplementary Fig. 1).

For the extension assay, we used unlabeled DNA substrate of the same sequence tested above. To visualize the extended substrate as the telomeric repeats are added, we prepared fluorescently (Cy3, green) labeled DNA probe which bears complementary sequence to the TTAGGG repeats. The length was optimized to 15 nucleotides (5'-CCCTAACCCTAACCC) which corresponds to two and a half repeats of TTAGGG such that the probe will remain bound stably to the newly synthesized DNA on the extended overhang. The length of the probe was decided on the basis of promoting annealing before potential G-quadruplex formation while achieving stable binding with the product for reliable detection of extension. Second, the concentration of the probe was determined to maximize rapid and efficient annealing while minimizing the fluorescence background signal. Therefore, the stepwise increase of one, two and three fluorophore intensities corresponds to extension of three, six and nine repeats, respectively (Fig. 1A). We initiated the extension by adding $10 \mathrm{nM}$ fluorescent probe and $500 \mu \mathrm{M}$ dNTP (deoxy-nucleotide phosphates) to substrate bound telomerase. When the buffer devoid of dNTP was applied, we obtained single molecule traces that display a small increase in intensity due to the background signal from the fluorescent probe in the buffer (Fig. 1B). The signal remains constant over time as expected from the lack of extension activity in the absence of dNTP. In contrast, when dNTP containing buffer was applied, we observed a stepwise increase in fluorescence, indicating a progressive extension of the telomeric overhang (Fig. 1C). Over the course of 15-30 minutes of measurement, we observed the appearance of more fluorescence spots and an increase in their intensity, reflecting more initiated extension events and longer extended products generated per telomerase, respectively (Supplementary Fig. 2a).

While the number of steps observed in single molecule traces can be translated to the length of the extended telomere, we found that the accuracy of this measurement was compromised by the occasional blinking and photobleaching of signals during the extension period (Supplementary Fig. 2b). To account for this, we used an alternative approach of counting photobleaching steps of the accumulated probes remaining annealed to the elongated product after 30 minutes of an active extension period (Fig. 1D, Supplementary Fig.
3). This was feasible because the extended products remained bound to telomerase for a sufficiently long period (over several minutes). We reasoned that the total length of extension or processivity estimated by this method may be underestimated due to the following factors. First, some fraction of the extended overhangs may not be fully accessible to the probe binding due to the occupancy by telomerase. Second, the probe may not stably bind to the last few repeats of the extended overhang because of its length (15 nt), which requires at least three repeats. To address these concerns, we performed gel electrophoresis analysis of ensemble telomerase reactions which visualize radioactively labeled extension products, to demonstrate that the longest extension product visible in the gel is comparable to the estimates from the single molecule assay (12-14 repeats) (Supplementary Fig. 4a, Fig. 2E). Additionally, we performed EMSA analysis to measure the binding of the fluorescent probe to various lengths of telomeric overhangs and show that the probe binding produces one tight shifted band in a length dependent manner, suggesting an efficient binding of probes to all lengths of overhangs tested (Supplementary Fig. 4b). We also compared the extension measured by gel electrophoresis and the single molecule assay. For this comparison, we used ImageQuant to quantitate the gel band intensities and calculated the fraction of molecules extended 3 , 6, 9 and 12 repeats, and then plotted them adjacent to the single molecule extension results (Supplementary Fig. 4c).

Telomerase extension rate and length display dNTP concentration dependence. Next, we examined the extension activity in 5 to $500 \mu \mathrm{M}$ concentration of dNTP. The representative single molecule traces collected at 5,50 and $500 \mu \mathrm{M}$ dNTP show that the rate of extension increases proportionally to the $\mathrm{dNTP}$ concentration (Fig. 2A). We termed the first lag period "activation" time which likely entails a conformational change of telomerase followed by the extension of three repeats. The subsequent periods are the "extension" time required for extending three repeats of overhang. The dwell times $(\delta \mathrm{t})$ collected from 50-100 molecules per dNTP concentration for both activation and extension show a dependence on dNTP (Fig. 2B). The estimated extension rate of $1 \mathrm{nt} / \mathrm{sec}$ $(60 \mathrm{nt} / \mathrm{min})$ matches closely with the recently published data ${ }^{19}$. Further, the total length of the extended products also exhibited dNTP concentration dependence (Fig. 2C). Taken together, our
A

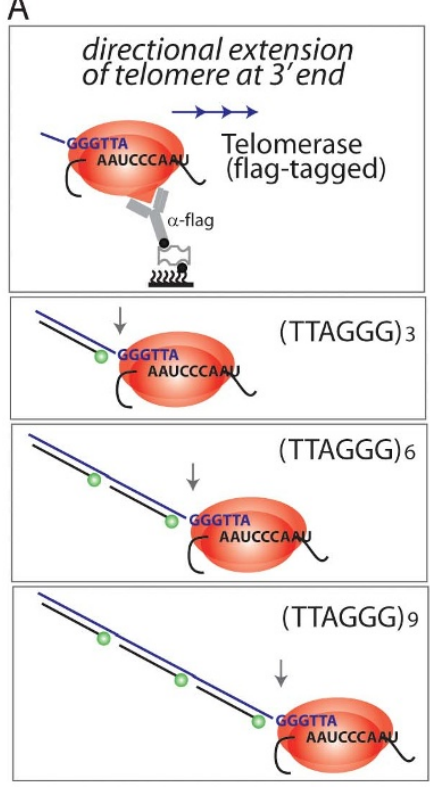

B

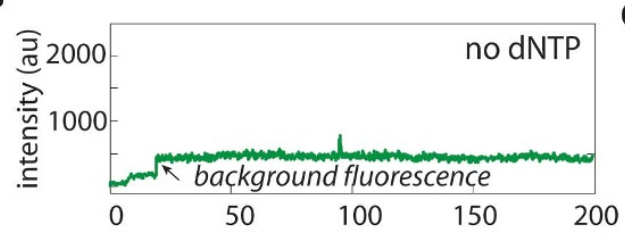

$\mathrm{D}$
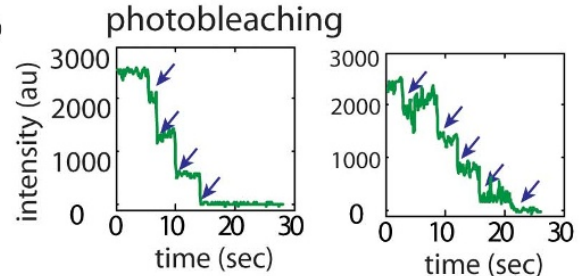

C
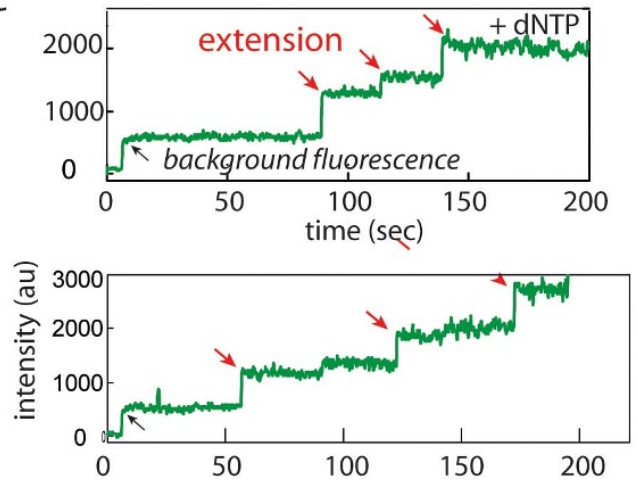

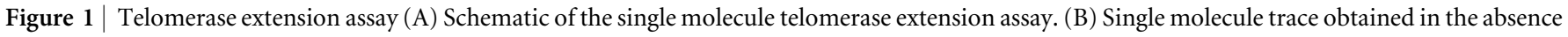
of dNTP. (C) Single molecule trace obtained in the presence of dNTP. (D) Quantitation of extension by counting photobleaching steps. 

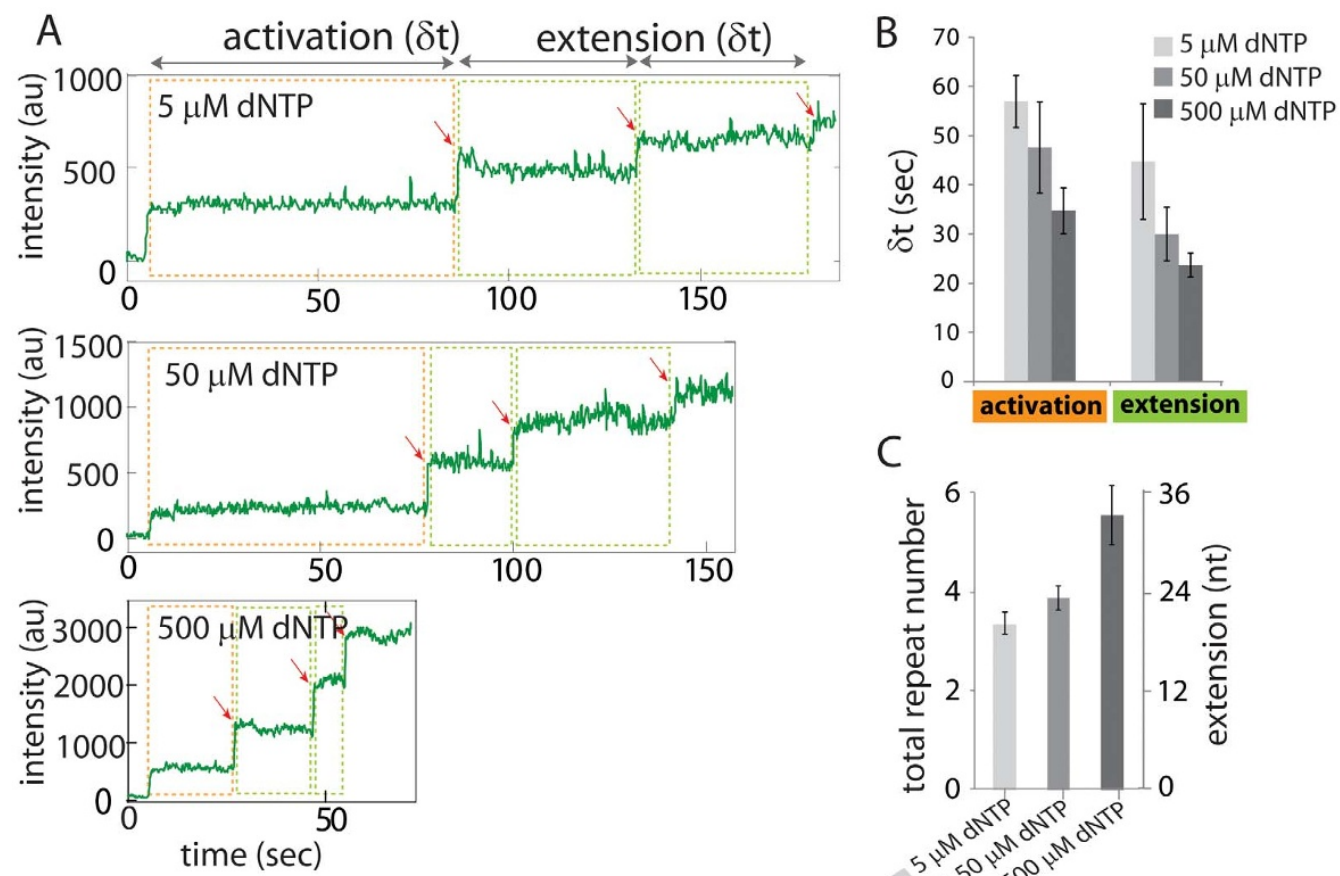

C

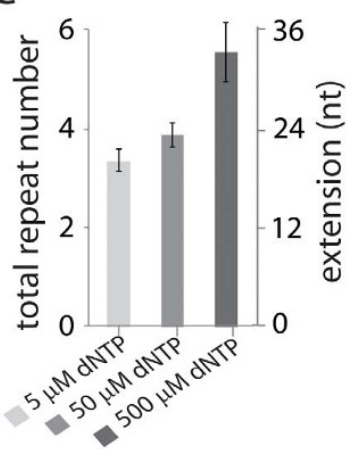

Figure 2 $\mid$ dNTP dependence of telomerase activity (A) Single molecule traces collected at different dNTP concentrations. (B) Dwell time analysis of activation and extension times taken at different dNTP concentrations (88, 43 and 91 molecules used for 5, 50 and $500 \mu \mathrm{M}$ dNTP respectively). (C) Total length of extension products measured at varying dNTP concentrations (approximately 350 molecules used for each dNTP condition).

results indicate that optimal dNTP concentration is needed to prevent delayed extension and the early termination that generates short overhang products.

POT1-TPP1 induces faster and longer extension. Two shelterin components, POT1 and TPP1 as a complex, act as a processivity factor for telomerase, generating substantially longer telomere overhangs $^{15}$. To gain insight about the role of the processivity factor, we added POT1-TPP1 to our extension assay (Fig. 3A). The majority of single molecule traces obtained in this experiment exhibited a significantly accelerated extension followed by a rapid dissociation of the extended product, reflected as a rapid stepwise increase and an instantaneous drop in fluorescence, respectively (Fig. 3B). Some show more than one burst of extension from the same telomerase (from the same fluorescence spot), reflecting more than one round of extension by a single unit of telomerase (Supplementary Fig. 5). Due to the immediate dissociation observed in the presence of POT1-TPP1, we estimated the total length of the extension by counting the stepwise increase in signal (Fig. 3C). The dwell time $(\delta \mathrm{t})$ analysis indicates that the initial activation time is comparable with and without POT1-TPP1, but the extension time is approximately six times faster in the presence of the POT1-TPP1 (Fig. 3D). In addition, the total length of extension product is substantially increased in the presence of POT1-TPP1 (Fig. 3E). We also show that TPP1 alone does not influence the telomerase extension activity (Supplementary Fig. 6).

Our data indicate that POT1-TPP1 increases the processivity of telomerase by accelerating the extension and by promoting synthesis of longer telomeric overhang lengths. The accelerated extension is not due to enhanced unfolding of G-quadruplex (GQ) by POT1TPP1 since an alternate DNA substrate, G7 (seven repeats of TTAGGG) which is capable of forming GQ yields the similar level of difference in the absence and presence of POT1-TPP1 (Supplementary Fig. 7). If the unfolding by POT1-TPP1 is the primary mechanism, the activation time should be reduced in the presence of POT1-TPP1, yet it remains approximately the same. In contrast to POT1-TPP1, the addition of POT1 alone greatly reduced the telomerase binding and activity. The small number of traces (2-3 per imaging area) obtained in the POT1 condition resemble that of telomerase extension shown in Figure 1C, suggesting that they represent telomeric template DNA without POT1 bound. The average number of extension events plotted for telomerase alone, telomerasePOT1 and the telomerase-POT1-TPP1 complex show that POT1 inhibits, whereas POT1-TPP1 enhances the binding and activity of telomerase (Fig. 3F). Further, the average length of extended products also shows that POT1-TPP1 promotes telomerase to produce a longer product whereas POT1 induces production of a shorter telomeric overhang (Fig. 3G). This finding is in agreement with a previous report ${ }^{20}$.

POT1-TPP1 induces dynamic fluctuation of telomeric DNA while engaged with telomerase. We showed that POT1-TPP1 slides on the telomeric overhang thereby inducing dynamic conformational change of the telomeric overhang ${ }^{21}$. We noticed that some single molecule traces displayed a rapidly fluctuating signal indicative of the protein dynamics that induces periodic fluorescence enhancement $^{22}$ (Fig. 4A, B). Briefly, the fluorescence intensity becomes brighter (2-3 fold) when the protein approaches the vicinity of the dye $^{23}$. Therefore, the fluctuating intensity indicates that the distance between the dye and protein undergoes a continuous change. The signal fluctuation does not interfere with measurement of extension because the intensity increase due to annealing of fluorescent probe is substantially larger than the range of fluctuation. Such signal fluctuation was only observed in the presence of POT1-TPP1, not when telomerase extends in the absence of POT1-TPP1 (Fig. 1C, 2A). This is reminiscent of the sliding activity of POT1-TPP1 near $3^{\prime}$ end of telomeric overhang we reported previously ${ }^{21}$. To test if POT1TPP1 generates similar conformational dynamics on telomeric DNA while engaged with telomerase, we added a FRET (Cy3 and Cy5) labeled DNA construct which reports on the conformational changes within the telomeric overhang. The single molecule traces display a stable FRET in the absence of POT1-TPP1 (Fig. 4C-E, 
A

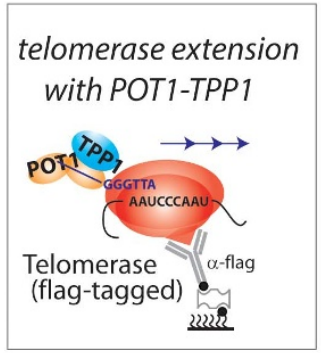

B
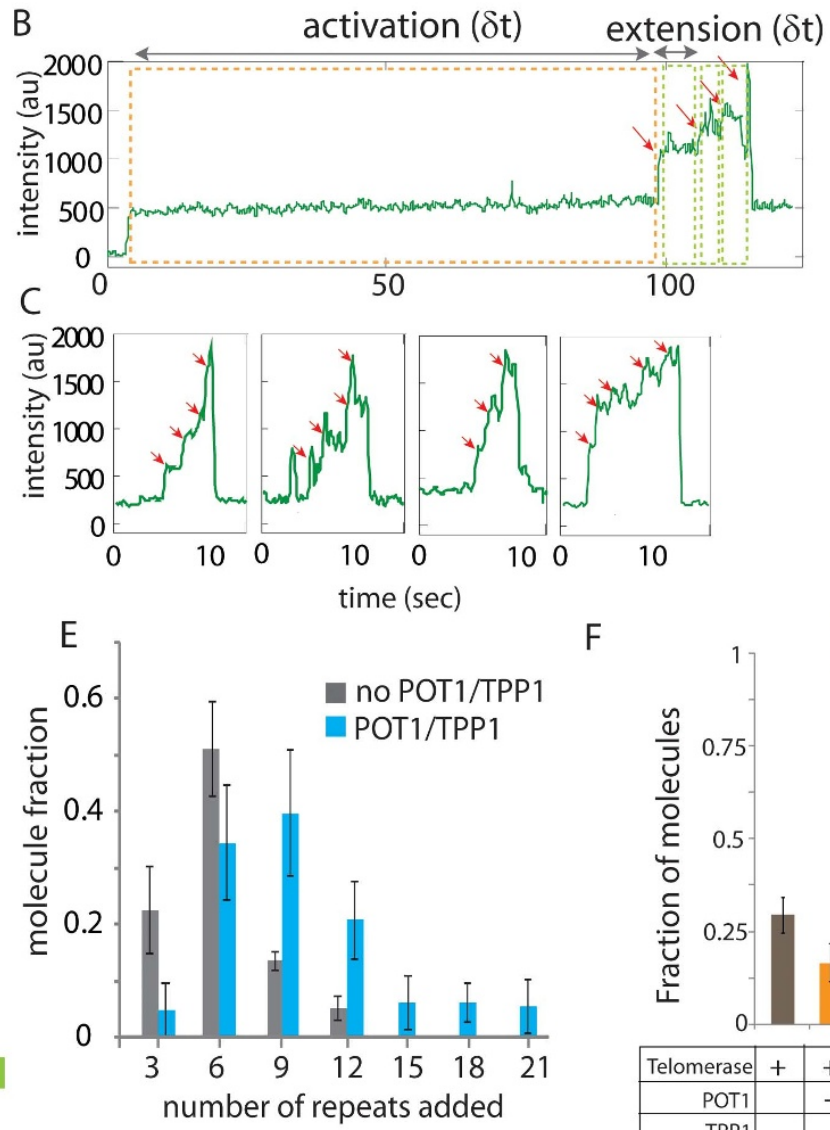

$\mathrm{F}$

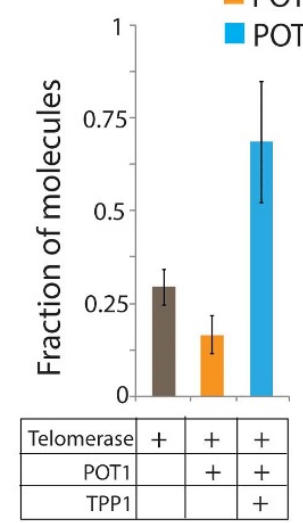

no POT1/TPP1

POT1

POT1/TPP1

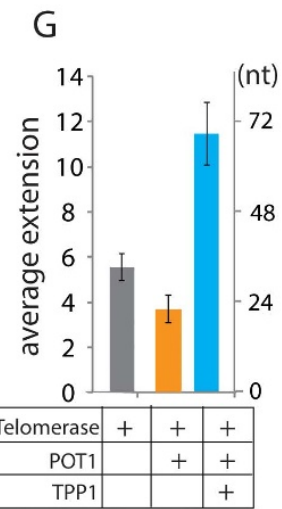

Figure $3 \mid$ POT1-TPP1 effect on telomere extension (A) Schematic of single molecule telomerase extension assay with POT1-TPP1. (B) Single molecule trace obtained in the presence of POT1-TPP1. (C) Rapid extension induced by POT1-TPP1. (D) Dwell time analysis of activation and extension times with and without POT1-TPP1 (collected from 198 molecules). (E) Distribution of extended products with and without POT1-TPP1. (F) Total number of extension events with POT1 vs. with POT1-TPP1 (337 molecules used). (G) Average length of extended products with POT1 vs. with POT1TPP1.

top). When POT1-TPP1 was introduced to form a complex with telomerase, we obtained rapidly fluctuating FRET traces (Fig. 4C$\mathrm{E}$, bottom). Based on our data, it is plausible to predict that the dynamics, which likely involve sliding movements of POT1-TPP1, may contribute to accelerating the telomerase extension activity while also providing the enhanced processivity. One possibility is that the sliding of POT1-TPP1 promotes faster annealing of the probe by resolving possible G-quadruplex structures. Therefore, we tested the probe binding to various lengths of telomeric overhangs (3.5, 6 and 7 repeats of TTAGGG) in the absence and presence of POT1-TPP1. The comparable rates obtained for all conditions confirm that POT1-TPP1 does not accelerate probe annealing (Supplementary Fig. 8). Therefore, the faster rate of extension we obtain here is due to the activity of POT1-TPP1 that accelerates telomerase extension. We note that the condition applied here (Fig. 4C-E) is not designed for extension, but for probing molecular interaction of the POT1-TPP1-telomerase complex and telomeric DNA. The end-labeled DNA used here does not permit elongation.

\section{Discussion}

The single molecule approach presented here has limitations but provides complementary information to the bulk experiment by providing single enzyme activity and improved kinetic parameters. For example, this assay likely underestimates the processivity of telomerase possibly due to limited binding of the probe. That is the extended length may be longer than what we observe by the probe binding due to the length of the probe and the $3^{\prime}$ end occupied by the telomerase. The major advantage of our single molecule platform is its unique ability to resolve kinetic rates of extension by individual units of telomerase in a digital manner. This allowed us to directly monitor and assess the impact of POT1-TPP1 on telomere extension. Telomerase extension can be divided into three phases; (i) initial activation involving a conformational change of telomerase, (ii) elongation of a telomere overhang by chemical addition of dNTP, (iii) translocation of telomerase entailing physical movement of telomerase for the subsequent elongation. The faster rate of extension induced by POT1-TPP1 that we obtained may arise from accelerating either the chemical (ii) or physical (iii) step. We rule out the activation step since we showed that it remains approximately the same in the absence or presence of POT1-TPP1 (Fig. 2D). Although we cannot decouple the chemical and physical step, we favor the model that POT1-TPP1 enhances the physical step by inducing a faster translocation of telomerase by sliding behind it. This is based on our previous and current observation of the dynamic activity of POT1-TPP $1^{21}$. For example, the continual sliding of POT1-TPP1 may serve to propel the telomerase forward at a faster rate.

The longer length of telomere overhangs generated in the presence of POT1-TPP1 is in agreement with previous findings ${ }^{15}$. Despite the longer length, our results indicate that the actual contact time between telomerase and the extended product is greatly reduced in the presence of POT1-TPP1 as evidenced by the rapid disappearance of the fluorescence signal (Fig. 3B, C). In this regard, the increased processivity by POT1-TPP1 is dominated by the kinetic effect. If the accelerated extension is primarily due to the faster translocation induced by POT1-TPP1, it is likely that the major rate limiting step 

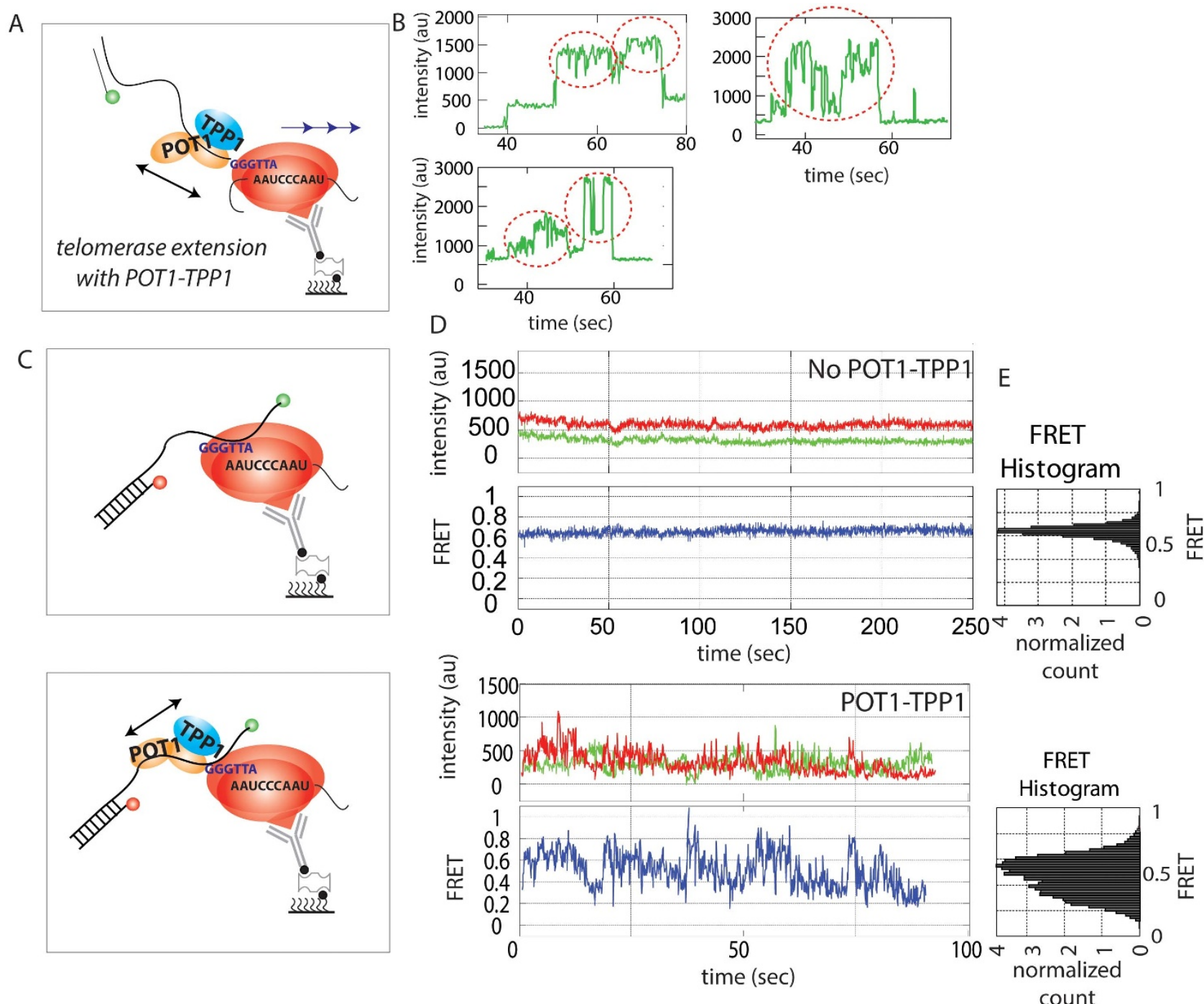

count

FRET

Histogram

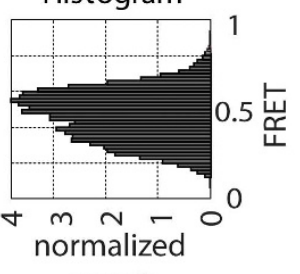

count

Figure 4 Sliding of POT1-TPP1 on telomerase-bound overhangs (A) Schematic of telomerase extension in the presence of POT1 and TPP1 (B) Single molecule traces that displays intensity fluctuations indicative of dynamic movement of POT1-TPP1 while telomerase is extending. (C) Schematic of applying FRET DNA to telomerase in the absence (top) and presence (bottom) of POT1-TPP1. (D) Single molecule FRET trace obtained in the absence (top) and presence (bottom) of POT1-TPP1. (E) FRET histogram generated in the absence (top) and presence (bottom) of POT1-TPP1.

in telomerase extension is the physical step even in the absence of POT1-TPP1. This also means that the elongation or the chemical step is inherently a fast process. Looking ahead, our detection platform can be useful for measuring kinetic parameters for mutated telomerase found in cancer cells or genetic disorders, testing the effect of telomerase inhibitor drugs and investigating the impact of shelterin components and accessory factors on telomerase activity.

\section{Methods}

Buffers. All experiments were conducted using telomerase extension buffer $50 \mathrm{mM}$ Tris-Cl pH8, $50 \mathrm{mM} \mathrm{KCl}$ and $1 \mathrm{mM} \mathrm{MgCl}$. The addition of $0.1 \mathrm{mg} / \mathrm{ml}$ bovine serum albumin (New England Biolabs) was used as wash buffer to minimize nonspecific binding of proteins to the surface. Unbound antibody was removed from the channel by washing with wash buffer between sample additions.

Oligonucleotides were purchased from IDT (Table 1). Single stranded DNA was used for the telomerase extension assays, while partial duplex substrates were used for the FRET experiment and for the gel shift assays. For the FRET constructs, partial duplex telomeric DNA constructs were prepared by mixing a Cy3 sequence with the Cy5-biotin sequence at a molar ratio of 1:1 in $20 \mathrm{mM}$ Tris- $\mathrm{HCl} \mathrm{pH} \mathrm{7.5,50} \mathrm{mM} \mathrm{NaCl}$ and incubating at $95^{\circ} \mathrm{C}$ and then slowly cooled to room temperature. For the gel shift assay experiment, $1: 1.5$ of unlabeled telomeric sequence and the Cy5 bottom strand $\mathrm{Cy} 5$ sequence were annealed.

POT1 and TPP1N protein purification. Recombinant human POT1 protein was purified using a baculovirus/insect cell expression system as described previously ${ }^{24}$.
The hexahistidine Sumo-tagged TPP1N (amino acids 89-334) construct was kindly provided by Dr. Ming Lei (University of Michigan). Expression was induced with $0.1 \mathrm{mM}$ isopropyl 1-thio-b-D-galactopyranoside in Escherichia coli BL21 (DE3) pLysS cells, and the protein was purified as previously described ${ }^{24}$.

Telomerase overexpression. Telomerase was overexpressed using a modification of an established protocol ${ }^{25}$. Cell lysate containing recombinant telomerase was reconstituted in HEK293T cells by overexpressing hTR and $3 \times$ FLAG tagged hTERT genes in pBS-U1-hTR and pVan107 respectively (generous gifts of Gaël Cristofari and Tom Cech). Cells were grown to $90 \%$ confluency containing DMEM medium (Gibco), supplemented with $10 \%$ FBS (Gibco) and $1 \%$ Pen Streb (Gibco) at $37^{\circ} \mathrm{C}$ and $5 \% \mathrm{CO}_{2}$. Cells were then transfected with $10 \mu \mathrm{g}$ of pBS-U1-hTR plasmid and $2.5 \mu \mathrm{g}$ pVan 107 plasmid diluted in $625 \mu$ Opti-MEM (Gibco) using $25 \mu$ lipofectamine 2000 (Invitrogen) diluted in $625 \mu \mathrm{l}$ Opti-MEM according to the manufacturer's instruction. Cells were allowed to grow for 48 hours post-transfection. Then, they were trypsinized and washed with phosphate buffered saline and lysed using $500 \mu \mathrm{l}$ Chaps lysis buffer (10 mM Tris-HCl, $1 \mathrm{mM} \mathrm{MgCl2,} 1 \mathrm{mM}$ EDTA, 0.5\% CHAPS, $10 \%$ Glycerol, $5 \mathrm{mM} \beta$-Mercaptoethanol, $120 \mathrm{U}$ Promega RNasin plus, $1 \mu \mathrm{g} / \mathrm{ml}$ pepstatin, aprotinin, leupeptin, chymostatin, and $1 \mathrm{mM}$ AEBSF). The cell lysate was then flash frozen in liquid nitrogen and stored at $-80^{\circ} \mathrm{C}$.

Single molecule setup. Single molecule fluorescence experiments were carried out on quartz slides (Finkenbeiner). Quartz slides and coverslips were coated with polyethylene glycol $(\mathrm{PEG})^{26}$. The slides and coverslips were treated with methanol, acetone, potassium hydroxide, burned, treated with aminosilane, and then coated with a mixture of 97\% mPEG (m-PEG-5000, Laysan Bio, Inc.) and 3\% biotin PEG (biotin-PEG-5000, Laysan Bio, Inc). A homemade wide-field prism-type TIRF setup 
G3TTAG (for sliding) Telomeric (for gel shift) 18 mer bottom (for sliding and gel shift) Cy3-probe
TGG CGA CGG CAG CGA GGC TTA GGG TTA GGG TTA GGG TTA GG/3Cy3/

TGG CGA CGG CAG CGA GGC (TTA GGG)n, where $\mathrm{n}$ is the number of repeats

5Cy5/GCC TCG CTG CCG TCG CCA annealed to all the Cy3 sequence listed above) Cy3-CCCTAACCCTAACCC was used with a solid-state $532 \mathrm{~nm}$ laser to generate an evanescent field of illumination. Cy3 signals were collected using a 630-nm dichroic mirror and sent to a charge-coupled device (CCD) camera.

smFRET Telomerase-DNA Co-localization study. Single molecule pull-down method was used to pull down the telomerase complex from over expressed telomerase cell lysate ${ }^{17}$. As described previously, telomerase was isolated onto the biotinylated PEG coated quart surface in the following order: Streptavidin, 1:50 antiflag antibody (Sigma, mouse M2, F9291), 1:200 cell lysate, and ss-Cy3 DNA [preaccepted at Structure]. Next, $1: 100$ hTERT antibody (Abcam, rabbit monoclonal) and secondary antibody labeled with Alexa 647 (Jackson Immunoresearch, donkey anti-rabbit) was added to colocalize the DNA with the telomerase. An alternating excitation pattern (first with $532 \mathrm{~nm}$ and then at $568 \mathrm{~nm}$ ) was used to excite the molecules and colocalize the Cy3-DNA and the Alexa647-telomerase, as shown in Supplemental Figure 1. To avoid false colocalization, samples were immobilized an optimal surface density (maximum 200 molecules in one field of view).

Single telomerase fluorescence extension assay. Telomerase was immobilized to the surface through single molecule pull-down methods from overexpressed cell. Biotinylated anti-flag-antibodies (1:50 Sigma, mouse M2, F9291) were added and then cell lysate was added at a 100 fold protein excess over the antibody. Next, the substrate $10 \mathrm{nM}$ ssG3 DNA was incubated without or with proteins (400 nM POT1 or 400 nM POT1-TPP1).

Fluorescence intensities of molecules were recorded with a time resolution of 50$100 \mathrm{~ms}$ as a stream of imaging frame, and analyzed with scripts written in interactive data language to give fluorescence intensity time trajectories of individual molecules. Single molecule imaging was initiated by flowing in $500 \mu \mathrm{M}$ (or otherwise noted) dNTP and $10 \mathrm{nM} \mathrm{Cy3}$ labeled probe in the imaging buffer containing an oxygen scavenging system $(0.8 \mathrm{mg} / \mathrm{ml}$ glucose oxidase, $0.625 \%$ glucose, $3 \mathrm{mM} 6$ - hydroxy2,5,7,8-tetramethylchromane-2-carboxylic (Trolox), and $0.03 \mathrm{mg} / \mathrm{ml}$ catalase). This movie captures the first moment of $\mathrm{Cy} 3$ probe binding and successive probe binding events that follow. Then, single molecule movies were obtained for 30 minutes, with each region being imaged for $\sim 200$ seconds at a time. All experiments and measurements were carried out at room temperature $(23 \mathrm{C} \pm 1 \mathrm{C})$.

Photobleaching analysis. After the extension assay of 30-40 minutes, the molecules were rinsed with wash buffer and subjected to photobleaching by strong laser illumination. The number of photobleaching steps in each trace was manually counted. Traces were manually classified as having 1-7 bleaching steps, and discarded if no clear bleaching steps could be identified. Separate counts were maintained for each movie, and at least 200 extended molecules were analyzed for each sample.

smFRET data analysis. Traces of molecules were viewed and analyzed with scripts written in Matlab. Histograms were generated using over 200 molecules collected and were fit to Gaussian distributions using Origin 8.0, with the peak position left unrestrained. Dwell times were collected by measuring the time before the probe binds after dNTP was added (activation time) and the time between each additional probe binding (extension time). Dwell times are an average of all the molecules, with the standard error of the mean as error bars.

Gel mobility shift assay. $10 \mathrm{nM}$ partial duplex telomeric overhang (TGGCGACGGCAGCGAGGC (TTA GGG) annealed to Cy5-bottom strand as stated above, with $\mathrm{n}$ as the number of repeats) was prepared. Cy3 complimentary strand (Cy3-CCCTAACCCTAA) was incubated for 15 minutes at room temperature in the telomerase extension buffer. The reaction mixture was loaded and ran on a $12 \%$ acrylamide gel at $200 \mathrm{~V}$ for 35 minutes with $1 \times$ TBE running buffer. Cy5 was imaged in the gel shown in Supplementary Figure 4.

1. Greider, C. W. \& Blackburn, E. H. Identification of a specific telomere terminal transferase activity in Tetrahymena extracts. Cell 43, 405-413 (1985).

2. Cech, T. R. Beginning to understand the end of the chromosome. Cell 116, 273-279 (2004).

3. Blasco, M. A. Telomeres and human disease: ageing, cancer and beyond. Nat Rev Genet 6, 611-622 (2005).

4. Meier, U. T. The many facets of H/ACA ribonucleoproteins. Chromosoma 114, 1-14 (2005).

5. Mitchell, J. R., Wood, E. \& Collins, K. A telomerase component is defective in the human disease dyskeratosis congenita. Nature 402, 551-555 (1999).

6. Mochizuki, Y., He, J., Kulkarni, S., Bessler, M. \& Mason, P. J. Mouse dyskerin mutations affect accumulation of telomerase RNA and small nucleolar RNA, telomerase activity, and ribosomal RNA processing. Proc Natl Acad Sci US A 101, 10756-10761 (2004).

7. Venteicher, A. S. et al. A human telomerase holoenzyme protein required for Cajal body localization and telomere synthesis. Science 323, 644-648 (2009).

8. Morin, G. B. The human telomere terminal transferase enzyme is a ribonucleoprotein that synthesizes TTAGGG repeats. Cell 59, 521-529 (1989).

9. Ohyashiki, K. et al. Cytological detection of telomerase activity using an in situ telomeric repeat amplification protocol assay. Cancer Res 57, 2100-2103 (1997)

10. Wright, W. E., Shay, J. W. \& Piatyszek, M. A. Modifications of a telomeric repeat amplification protocol (TRAP) result in increased reliability, linearity and sensitivity. Nucleic Acids Res 23, 3794-3795 (1995).

11. Kim, N. W. \& Wu, F. Advances in quantification and characterization of telomerase activity by the telomeric repeat amplification protocol (TRAP). Nucleic Acids Res 25, 2595-2597 (1997).

12. Reed, J., Gunaratnam, M., Beltran, M., Reszka, A. P., Vilar, R. \& Neidle, S. TRAPLIG, a modified telomere repeat amplification protocol assay to quantitate telomerase inhibition by small molecules. Analytical biochemistry 380, 99-105 (2008).

13. Zhou, X. \& Xing, D. Assays for human telomerase activity: progress and prospects. Chemical Society reviews 41, 4643-4656 (2012).

14. Ren, X. et al. Analysis of human telomerase activity and function by two color single molecule coincidence fluorescence spectroscopy. J Am Chem Soc 128, 4992-5000 (2006).

15. Wang, F. et al. The POT1-TPP1 telomere complex is a telomerase processivity factor. Nature 445, 506-510 (2007).

16. Latrick, C. M. \& Cech, T. R. POT1-TPP1 enhances telomerase processivity by slowing primer dissociation and aiding translocation. EMBO J 29, 924-933 (2010).

17. Jain, A. et al. Probing cellular protein complexes using single-molecule pull-down. Nature 473, 484-488 (2011).

18. Hwang, H. et al. Telomeric Overhang Length Determines Structural Dynamics and Accessibility to Telomerase and ALT-Associated Proteins. Structure 22, 842-53 (2014).

19. Xi, L. \& Cech, T. R. Inventory of telomerase components in human cells reveals multiple subpopulations of hTR and hTERT. Nucleic Acids Res 42, 8565-8577 (2014).

20. Kelleher, C., Kurth, I. \& Lingner, J. Human protection of telomeres 1 (POT1) is a negative regulator of telomerase activity in vitro. Mol Cell Biol 25, 808-818 (2005).

21. Hwang, H., Buncher, N., Opresko, P. L. \& Myong, S. POT1-TPP1 Regulates Telomeric Overhang Structural Dynamics. Structure 20, 1872-80 (2012).

22. Hwang, H., Kim, H. \& Myong, S. Protein induced fluorescence enhancement as a single molecule assay with short distance sensitivity. Proc Natl Acad Sci US A 108, 7414-7418 (2011).

23. Myong, S. et al. Cytosolic viral sensor RIG-I is a $5^{\prime}$-triphosphate-dependent translocase on double-stranded RNA. Science 323, 1070-1074 (2009).

24. Sowd, G., Wang, H., Pretto, D., Chazin, W. J. \& Opresko, P. L. Replication protein A stimulates the Werner syndrome protein branch migration activity. J Biol Chem 284, 34682-34691 (2009).

25. Cristofari, G. \& Lingner, J. Telomere length homeostasis requires that telomerase levels are limiting. EMBO J 25, 565-574 (2006).

26. Roy, R., Hohng, S. \& Ha, T. A practical guide to single-molecule FRET. Nature methods 5, 507-516 (2008).

\section{Acknowledgments}

We thank Justin Lormand from the Opresko lab for preparing cell lysate and conducting the gel based telomerase assay and Myong group members for helpful discussions. Support for this work was provided by NIH Director's New Innovator Award (343 NIH 1 DP2 GM105453) and American Cancer Society (Research Scholar Grant;

RSG-12-066-01-DMC) for S.M., NIH National Cancer Institute (1F30CA174323-01) and Linda Su-Nan Chang Sah Doctoral Fellowship for H.H. and NIH grant R01ES022944 and the David Scaife Foundation for P.L.O.

\section{Author contributions}

H.H. performed experiments, analyzed data and S.M. planned the research and wrote the paper, P.O. planned the research, provided telomerase cell lysate and edited the paper.

\section{Additional information}

Supplementary information accompanies this paper at http://www.nature.com/ scientificreports 
Competing financial interests: The authors declare no competing financial interests.

How to cite this article: Hwang, H., Opresko, P. \& Myong, S. Single-molecule real-time detection of telomerase extension activity. Sci. Rep. 4, 6391; DOI:10.1038/srep06391 (2014).

(c) (1) (2) This work is licensed under a Creative Commons Attribution-NonCommercialBY NC SA ShareAlike 4.0 International License. The images or other third party material in this article are included in the article's Creative Commons license, unless indicated otherwise in the credit line; if the material is not included under the Creative Commons license, users will need to obtain permission from the license holder in order to reproduce the material. To view a copy of this license, visit http:// creativecommons.org/licenses/by-nc-sa/4.0/ 